REVISTA MATEMÁTICA de la

Universidad Complutense de Madrid

Volumen 10, número 2: 1997

http://dx.doi.org/10.5209/rev_REMA.1997.v10.n2.17438

\title{
Some properties of basic sequences in Banach spaces.
}

\author{
Manuel VALDTVIA*
}

\begin{abstract}
Summary
Some classes of basic sequences in Banach spaces are studied. We show in particular that if $X$ is a Banach space with separable dual $X^{*}$ and $U \subset V$ are norming closed subspaces of $X^{*}$, then there is a basic sequence $\left(x_{n}\right)$ in $X$ such that, if $\left[x_{n}\right]$ is the closed linear hull of $\left(x_{n}\right)$ and $\left[x_{n}\right]^{\perp}$ is the subspace of $X^{*}$ orthogonal to $\left[x_{n}\right], U+\left[x_{n}\right]^{\perp}=V$ and the weak ${ }^{*}$-closure of $U \cap\left[x_{n}\right]^{\perp}$ in $X^{*}$ coincides with $\left[x_{n}\right]^{\perp}$. This result, suggested by some problems in the quasi-reflexivity of Banach spaces, allows us to obtain some new results, as well as some already known ones, about this property. We also give here some results concerning Schauder basis in quotients of Banach spaces.
\end{abstract}

The linear spaces we shall be using in this paper are supposed to be defined over the field $\boldsymbol{K}$ of real or complex numbers. If $\boldsymbol{K}$ is real, then $\boldsymbol{H}$ is the field of rational numbers and, if $\boldsymbol{K}$ is complex, then $\boldsymbol{H}$ is the field formed by all numbers of the form $a+b i$ with $a$ and $b$ rationals. The set of positive integers will be denoted by $\mathbb{N}$. Unless otherwise stated, all Banach spaces considered here will be assumed to have infinite dimension. If $X$ is a Banach space, $\|\cdot\|$ will be its norm, $X^{*}$ is the Banach space conjugate of $X, X^{* *}$ is the conjugate of $X^{*}$ and $X^{* * *}$ the conjugate of $X^{* *}$. We identify $X$, in the usual way, with a subspace of $X^{* *}, B(X)$ is the closed unit ball of $X$ and $B(X)$ is the interior of $B(X)$, i.e., the open unit ball of $X$. If $x \in X$ and $u \in X^{*}$,

* Suppoorted in part by DGICYT PB91-0326.

Mathematics Subject Classification: 46B15.

Servicio Publicaciones Univ. Complutense. Madrid, 1997. 
we write $\langle x, u\rangle$ instead of $u(x)$. If $\left(x_{n}\right)$ is a sequence in $X$, by $\left[x_{n}\right]$ we mean its closed linear span. Similarly, if $\left(x_{m n}\right)$ is a double sequence in $X$, then $\left[x_{m n}\right]$ is its closed linear span. If $\left(x_{n}\right)$ is a basic sequence, then we put $x_{n}^{*}, n \in \mathbb{N}$, for the linear functionals in $\left[x_{n}\right]^{*}$ associated to the Schauder basis $\left(x_{n}\right)$ of $\left[x_{n}\right]$. If there is an integer $k$ greater or equal to zero such that $\left[x_{n}^{*}\right]$ has codimension $k$ in $\left[x_{n}\right]^{*}$, then the basic sequence $\left(x_{n}\right)$ is said to be $k$-shrinking; in particular, when $k=0,\left(x_{n}\right)$ is said to be shrinking. If the basic sequence $\left(x_{n}\right)$ has the property that for any given sequence $\left(a_{n}\right)$ in $\boldsymbol{K}$ such that

$$
\sup \left\{\left\|\sum_{j=1}^{n} a_{j} x_{j}\right\|: n \in \mathbb{N}\right\}<\infty
$$

the series $\sum_{j=1}^{\infty} a_{j} x_{j}$ is convergent, then $\left(x_{n}\right)$ is said to be boundedly complete. If the double sequence $\left(x_{m n}\right)$ of $X$ admits an ordering such that it is a basic sequence, then $x_{m n}^{*}, m, n \in N$, are the linear functionals of $\left[x_{m n}\right]^{*}$ associated to such a sequence. If $Y$ and $Z$ are closed subspaces, of finite or infinite dimension, of the Banach space $X, Y$ is said to be an orthogonal complement of $Z$ in $Z+Y$ if $Z \cap Y=\{0\}$ and in $Z+Y$ the projection onto $Z$ along $Y$ is continuous and has norm one.

For a given Banach space $X$, the weak* topology in $X^{*}$, or also $\sigma\left(X^{*}, X\right)$, is the topology of the pointwise convergence over $X$. If $Y$ is a subspace of $X^{*}, \sigma(X, Y)$ is the topology on $X$ of the pointwise convergence over $Y$; in particular, if $Y=X^{*}, \sigma\left(X, X^{*}\right)$ is the weak topology in $X$. If $A$ is a subset of $X, \operatorname{lin} A$ denotes the linear span of $A, \tilde{A}$ is the closure of $A$ in $X^{* *}$ for the weak* topology and $A^{\perp}$ is the subspace of $X^{*}$ orthogonal to $A$. If $B$ is a subset of $X^{*}$, then $\widetilde{B}$ will be its $\sigma\left(X^{*}, X\right)$-closure and $B_{\perp}$ its orthogonal subspace in $X$.

A Banach space $X$ is said to be quasi-reflexive if it is of finitecodimension in $X^{* *}$. In this case, if $k$ is its codimension, $X$ is said to be quasi-reflexive of order $k$. In particular, when $k=0, X$ is a reflexive space.

If $Z$ is a closed subspace of the Banach space $X$, the dimension of $Z$ being either finite or infinite, we identify in the usual manner $Z^{*}$ with $Z^{*} / Z^{\perp}$. Similarly, $(X / Z)^{*}$ identifies with $Z^{\perp}$. Let $V$ be a closed subspace of $X^{*}$. We put, for each $z$ of $Z$,

$$
|z|:=\sup \{|\langle z, u\rangle|: u \in B(V)\} .
$$


We say that $V$ is 1-norming (norming) with respect to $Z$ if $\|\cdot\|=|\cdot|$ in $Z(|\cdot|$ is an equivalent norm to $\|\cdot\|$ in $Z)$. If $Z=X$, we shall simply say that $V$ is a 1-norming (norming) subspace of $X^{*}$.

Let $\left(x_{n}\right)$ be a basic sequence in the Banach space $X$. We say that $\left(x_{n}\right)$ has Property $\mathrm{P}$, or that it is of the type $\mathrm{P}$, if inf $\left\{\left\|x_{n}\right\|: n \in \mathbb{N}\right\}>0$ and $\sup \left\{\left\|\sum_{j=1}^{n} x_{j}\right\|: n \in \mathbb{N}\right\}<\infty$. Then, $\left(\sum_{j=1}^{n} x_{j}\right)$ is also a basic sequence in $X$. On the other hand, $\left(x_{n}\right)$ is said to have Property $\mathrm{P}^{*}$, or that it is of the type $\mathrm{P}^{*}$, if

$$
\sup \left\{\left\|x_{n}\right\|: n \in N\right\}<\infty
$$

and the sequence $\left(v_{n}\right)$ such that

$$
v_{1}:=x_{1}, v_{2}:=x_{1}-x_{2}, \ldots, v_{n}:=x_{n-1}-x_{n}, \ldots
$$

is a Schauder basis in $\left[x_{n}\right]=\left[v_{n}\right]$. For example, the natural basis of $c_{0}$ is of the type $\mathrm{P}$, but it is not of the type $\mathrm{P}^{*}$. On the other hand, the natural basis of $l^{1}$ is of the type $P^{*}$ but not of the type $P$.

Properties $P$ and $P^{*}$ were introduced by Singer in [15]. The following result, to be found in [15] (see also [16, p. 311]), will be needed afterwards: a) $A$ basic sequence $\left(x_{n}\right)$ in the Banach space $X$ has property $P$ if and only if $\left(x_{n}^{*}\right)$ has property $P^{*}$.

For the next lemma we assume that $X$ is a Banach space and that $U$ is a finite-dimensional subspace of $X$. Let $B$ be a bounded absolutely convex subset of $X^{*}$ such that

$$
\|x\|=\sup \{|\langle x, u\rangle|: u \in B\}, \quad x \in U .
$$

We consider a weak* dense subset $S$ of $B$.

Lemma 1. Given $\varepsilon>0$, there is a finite subset $A$ of $S$ such that, if $u$ belongs to $B\left(X^{*}\right)$, then there is $v$ in $A$ such that

$$
\sup \{|\langle x, u-v\rangle|: x \in B(U)\} \leq \varepsilon .
$$

Proof. Let $\varphi$ be the canonical mapping from $X^{*}$ onto $X^{*} / U^{\perp}$. We know after (1) that the closure $M$ of $\varphi(B)$ in $X^{*} / U^{\perp}$ coincides with the closed unit ball of this space. For each $w$ in $M$, let $P_{w}$ be the open ball 
in $X^{*} / U^{\perp}$ centered at $w$ with radius $\frac{1}{2} \varepsilon$. Since $M$ is compact, there is a finite set $\left\{w_{1}, w_{2}, \ldots, w_{p}\right\}$ in $M$ such that $M$ is contained in $\cup_{j=1}^{p} P_{w_{j}}$. Now, since $\varphi(S)$ is dense in $M$, we may find, for each integer $j, 1 \leq j \leq p$, a vector $y_{j}$ in $S$ such that $\varphi\left(y_{j}\right)$ is in $P_{w_{j}}$. We set $A:=\left\{y_{j}: 1 \leq j \leq p\right\}$. Now, let $u$ be a vector in $B\left(X^{*}\right)$. We have that $\varphi(u)$ is in $M$ and thus it is in $P_{w_{*}}$ for some $s, 1 \leq s \leq p$. We write $v:=y_{s}$. Then

$$
\|\varphi(u)-\varphi(v)\| \leq\left\|\varphi(u)-w_{s}\right\|+\left\|w_{s}-\varphi(v)\right\|<\varepsilon
$$

and hence we have that (2) is satisfied.

In the next lemma, we obtain and extend some results of [1], [14], $[10]$ and [17].

Lemma 2. Let $\left(x_{m n}\right)$ be a double sequence in the Banach space $X$ such that

$$
\text { in } \left.f\left\|x_{m n}\right\|: n \in \mathbb{N}\right\}>0, \quad m \in \mathbb{N} \text {. }
$$

Let $\left(u_{n}\right)$ be a sequence in $X^{*}$ with $\left[u_{2 n}\right]$ 1-norming with respect to $\left[x_{m n}\right]$. If

$$
\lim _{n}\left\langle x_{m n}, u_{j}\right\rangle=0, \quad m, j \in \mathbb{N},
$$

then there is a subsequence $\left(y_{m n}\right)_{n=1}^{\infty}$ of $\left(x_{m n}\right)_{n=1}^{\infty}, m \in N$, such that

$$
y_{11}, y_{12}, y_{21}, \ldots, y_{1 n}, y_{2(n-1)}, \ldots, y_{(n-1) 2}, y_{n 1}, \ldots
$$

is a basic sequence in $X$ which is a subsequence of

$$
x_{11}, x_{12}, x_{21}, \ldots, x_{1 n}, x_{2(n-1)}, \ldots, x_{(n-1) 2}, x_{n 1}, \ldots
$$

such that, if $\varphi$ is the canonical mapping from $X^{*}$ onto $X^{*} /\left[y_{m n}\right]^{\perp}$, we have that

$$
\varphi\left(\stackrel{\circ}{B}\left(\left[u_{n} \mid\right)\right)=\varphi\left(\stackrel{\circ}{B}\left(\left[u_{2 n}\right]\right)\right)=\stackrel{\circ}{B}\left(\left[y_{m n}^{*}\right]\right) .\right.
$$

Proof. We may assume, with no loss of generality, that $\left\|x_{m n}\right\|=1$, $m, n \in \mathbb{N}$. Let

$$
F:=\operatorname{lin}\left\{u_{2 n}: n \in \mathbb{N}\right\} \text { and } G:=\operatorname{lin}\left\{u_{n}: n \in \mathbb{N}\right\} .
$$


We consider in $G \cap B\left(X^{*}\right)$ a countable dense subset $\left\{v_{m n}: m, n \in \mathbb{N}\right\}$ such that $\left\{v_{m(2 n)}: m, n \in \mathbb{N}\right\}$ be dense in $F \cap B\left(X^{*}\right)$. Clearly,

$$
\lim _{n}\left\langle x_{m n}, v_{p q}\right\rangle=0, \quad m, p, q \in \mathbb{N} .
$$

We endow the set of all pairs $m n$ with an order relation $\leq^{*}$ so that $p q<^{*} m n$ whenever $p+q<m+n$, or, $p+q=m+n$ and $p<m$. We take $0<\varepsilon_{m n}<1$ so that $\sum_{m, n} \varepsilon_{m n}<\infty$. We define now $y_{11}:=x_{11}$, $\rho(1,1):=11$. Proceeding by recurrence, we suppose that, for a given subindex $p q$, we have obtained from $\left\{x_{m n}: m, n \in \mathbb{N}\right\}$ the vectors $y_{m n}$, $m n \leq^{*} p q$ such that $y_{p q}=x_{\rho(p, q)}$. We then write

$$
Y_{p q}:=\operatorname{lin}\left\{y_{m n}: m n \leq * p q\right\}
$$

with the norm induced by that of $X$. Applying Lemma 1 we obtain a finite subset $A_{p q}$ of $\left\{v_{m(2 n)}: m, n \in \mathbb{N}\right\}$ containing $\left\{v_{m(2 n)}: m n \leq * p q\right\}$, so that, given any element $u$ of $B\left(X^{*}\right)$, there is an element $v$ in $A_{p q}$ such that if $z$ belongs to $B\left(Y_{p q}\right)$ then

$$
|\langle z, u-v\rangle| \leq \frac{1}{4} \varepsilon_{p q}
$$

We define

$$
B_{p q}:=A_{p q} \cup\left\{v_{m n}: m n \leq{ }^{*} p q\right\} .
$$

Let $r s$ be the successor of $p q$. We.put $p_{0} q_{0}$ for the greatest pair $m(2 n)$ for which $v_{m(2 n)}$ is in $A_{p q}$. It follows from (3) that there is a positive integer $n_{0}$ such that $p_{0} q_{0}<{ }^{*} r n_{0}, \rho(p, q)<{ }^{*} n_{0}$ and

$$
\left|\left\langle x_{r m}, w\right\rangle\right|<\frac{1}{8} \varepsilon_{p q}, \quad n \geq n_{0}, w \in \bigcup_{s t<<^{*} q} B_{s t} .
$$

We now define $y_{r s}:=x_{r n_{0}}, \rho(r, s):=r n_{0}$. Next we see a property of the vector $y_{r s}$. Take $z$ in $Y_{p q}$ with $z \|=1$. We find a vector $u$ in $X^{*}$ such that

$$
\|u\|=1, \quad\langle z, u\rangle=1 .
$$

We determine a vector $v$ in $A_{p q}$ satisfying (4). Then

$$
|\langle z, v\rangle| \geq|\langle z, u\rangle|-|\langle z, v-u\rangle| \geq 1-\frac{1}{4} \varepsilon_{p q} .
$$


Let $\lambda$ be an arbitrary element of $K$. If $|\lambda|>2$, then

$$
\left\|z+\lambda y_{r s}\right\| \geq|\lambda| \cdot\left\|y_{r s}\right\|-\|z\|>1,
$$

and, if $|\lambda| \leq 2$, then, having (5) in mind, it follows that

$$
\begin{gathered}
\left\|z+\lambda y_{r s}\right\| \geq\left|\left\langle z+\lambda y_{r s}, v\right\rangle\right| \geq|\langle z, v\rangle|-|\lambda| \cdot\left|\left\langle y_{r s}, v\right\rangle\right| \\
\geq 1-\frac{1}{4} \varepsilon_{p q}-2 \cdot \frac{1}{8} \varepsilon_{p q}=1-\frac{1}{2} \varepsilon_{p q}=1-\varepsilon_{p q}+\frac{\varepsilon_{p q}^{2}}{2 \varepsilon_{p q}} \\
\geq 1-\varepsilon_{p q}+\frac{\varepsilon_{p q}^{2}}{1+\varepsilon_{p q}}=\frac{1}{1+\varepsilon_{p q}} .
\end{gathered}
$$

Thus, for every $z$ in $Y_{p q}$ with $\|z\|=1$ and every $\lambda$ in $\boldsymbol{K}$, we have

$$
1 \leq\left(1+\varepsilon_{p q}\right)\left\|z+y_{r s}\right\| \text {. }
$$

Let's take now an arbitrary element $y$ of $Y_{p q}$ and $\alpha \in \mathbb{K}$. If $y \neq 0$, we apply (6) for $z:=\frac{y}{\|y\|}$ and $\lambda:=\frac{\alpha}{\|y\|}$, and so we obtain

$$
1 \leq\left(1+\varepsilon_{p q}\right)\left\|\frac{y}{\|y\|}+\frac{\alpha}{\|y\|} y_{r s}\right\| .
$$

Hence

$$
\|y\| \leq\left(1+\varepsilon_{p q}\right)\left\|y+\alpha y_{r s}\right\|, \quad y \in Y_{p q}, \quad \alpha \in \boldsymbol{K} .
$$

Clearly, $\left(y_{m n}: \leq^{*}\right)$ is a subsequence of $\left(x_{m n}: \leq^{*}\right)$. We see next that $\left(y_{m n}: \leq^{*}\right)$ is a basic sequence in $X$. We take two arbitrary subindices $p q$ and $r s$ so that $p q<^{*} r s$. Let $h k$ be the subindex immediately prior to rs. We take

$$
\left\{a_{i j} \in \mathbb{R}: i j \leq^{*} r s\right\} .
$$

Repeatedly making use of (7), it follows that

$$
\left\|\sum\left\{a_{i j} y_{i j}: i j \leq^{*} p q\right\}\right\| \leq\left(1+\varepsilon_{p q}\right) \ldots\left(1+\varepsilon_{h k}\right)\left\|\sum\left\{a_{i j} y_{i j}: i j \leq^{*} r s\right\}\right\|
$$

and therefore $\left(y_{m n}: \leq^{*}\right)$ is a basic sequence in $X$ whose basic constant $b$ is less or equal than $\prod_{m, n}\left(1+\varepsilon_{m n}\right)$. Besides, if we put $P_{m n}$ to denote the linear projections defined in $\left[y_{m n}\right]$ and associated to the basis $\left(y_{m n}: \leq^{*}\right)$, we have that

$$
\lim \left\{\left\|P_{m n}\right\|: \leq^{*}\right\}=1 .
$$


Let $\varphi$ stand for the canonical mapping from $X^{*}$ onto $X^{*} /\left|y_{m n}\right|^{\perp}$. We fix the subindex $p q$. In $\left[y_{m n}\right]^{*}=X^{*} /\left[y_{m n}\right]^{\perp}, \varphi\left(v_{p q}\right)$ is the sum of the series

$$
\sum\left\{\left\langle y_{m n}, \varphi\left(v_{p q}\right)\right\rangle y_{m n}^{*}: \leq^{*}\right\}
$$

for the weak* topology. For each subindex $m n$, let $m_{1} n_{1}$ be its successor. After (5) we obtain

$$
\left|\left\langle y_{m_{1} n_{1}}, w\right\rangle\right|<\varepsilon_{m n}, \quad w \in B_{m n}
$$

and therefore

$$
\begin{gathered}
\sum_{m_{1} n_{1} \geq^{*} p_{q}}\left\|\left\langle y_{m_{1} n_{1}}, \varphi\left(v_{p q}\right)\right\rangle y_{m_{1} n_{1}}^{*}\right\|=\sum_{m_{1} n_{1}{ }^{*} \geq p q}\left\|\left\langle y_{m_{1} n_{1}}, v_{p q}\right\rangle y_{m_{1} n_{1}}^{*}\right\| \\
\leq \sum_{m_{1} n_{1}{ }^{*} \geq p q}\left|\left\langle y_{m_{1} n_{1}}, v_{p q}\right\rangle\right| \cdot\left\|y_{m_{1} n_{1}}^{*}\right\| \leq 2 b \sum_{m_{1} n_{1}{ }^{*} \geq p q}\left|\left\langle y_{m_{1} n_{1}}, v_{p q}\right\rangle\right| \\
\leq 2 b \sum_{m, n} \varepsilon_{m n}<\infty,
\end{gathered}
$$

hence we deduce that the series (9) is absolutely convergent in the $\mathrm{Ba}$ nach space $\left[y_{m n}^{*}\right]$ and thus $\varphi\left(v_{p q}\right)$ belongs to $\left[y_{m n}^{*}\right]$, whence we conclude that $\varphi\left(\left[u_{n}\right]\right)$ is contained in $\left[y_{m n}^{*}\right]$. We take now a vector $y^{*}$ in $\left[y_{m n}^{*}\right]$ with $\left\|y^{*}\right\|=1$. Given $\varepsilon>0$, we find a positive integer $q$ such that

$$
\begin{gathered}
\left\|P_{1 q}\right\|<1+\frac{\varepsilon}{6(1+\varepsilon)}, \frac{b}{4} \sum\left\{\varepsilon_{m n}: 1 q<^{*} m n\right\}<\frac{1}{3} \varepsilon \\
\varepsilon_{1 q}<\frac{\varepsilon}{6(1+\varepsilon)},\left\|y^{*}-P_{1 q}^{*} y^{*}\right\|<\frac{1}{3} \varepsilon .
\end{gathered}
$$

We apply Hahn-Banach's theorem to obtain an element $w$ of $X^{*}$ that coincides with $P_{1 q}^{*} y^{*}$ on $\left[y_{m n}\right]$ and

$$
\|w\|=\left\|P_{1 q}^{*} y^{*}\right\| \leq\left\|P_{1 q}^{*}\right\|=\left\|P_{1 q}\right\|<1+\frac{\varepsilon}{6(1+\varepsilon)} .
$$

Then

$$
\left\|\left(1+\frac{\varepsilon}{6(1+\varepsilon)}\right)^{-1} w\right\|<1
$$


and thus we know, after (4), that there is $v$ in $A_{1 q}$ such that, if $y$ belongs to $B\left(Y_{1 q}\right)$, then we have

$$
\left|\left\langle y,\left(1+\frac{\varepsilon}{6(1+\varepsilon)}\right)^{-1} w-v\right\rangle\right| \leq \varepsilon_{1 q}
$$

and, consequently,

$$
\begin{aligned}
|\langle y, w-v\rangle| \leq\left|\left\langle y, w-\left(1+\frac{\varepsilon}{6(1+\varepsilon)}\right)^{-1} w\right\rangle\right|+\left|\left(y,\left(1+\frac{\varepsilon}{6(1+\varepsilon)}\right)^{-1} w-v\right\rangle\right| \\
\leq\left(1-\left(1+\frac{\varepsilon}{6(1+\varepsilon)}\right)^{-1}\right)\|w\|+\varepsilon_{1 q} \\
<\frac{\varepsilon}{6(1+\varepsilon)}+\frac{\varepsilon}{6(1+\varepsilon)}=\frac{\varepsilon}{3(1+\varepsilon)} .
\end{aligned}
$$

Then, if we take $z$ in $B\left(\left[y_{m n} \mid\right)\right.$ and having in mind that $\left\|P_{1 q}\right\|^{-1} P_{1 q} z \in$ $B\left(Y_{1 q}\right)$, it follows that

$$
\begin{aligned}
\mid\left\langle z, P_{1 q}^{*}(\varphi(v)\right. & \left.\left.-y^{*}\right)\right\rangle|=|\left\langle z, P_{1 q}^{*}\left(\varphi(v)-P_{1 q}^{*} y^{*}\right)\right\rangle|=|\left\langle P_{1 q} z, \varphi(v)-P_{1 q}^{*} y^{*}\right\rangle \mid \\
& \leq\left\|P_{1 q}\right\| \cdot \mid\left\langle\left\|P_{1 q}\right\|^{-1} P_{1 q z, v-w\rangle \mid}\right. \\
& \leq\left(1+\frac{\varepsilon}{6(1+\varepsilon)}\right)\left|\left\langle\left\|P_{1 q}\right\|^{-1} P_{1 q} z, v-w\right\rangle\right| \\
& \leq\left(1+\frac{\varepsilon}{6(1+\varepsilon)}\right) \frac{\varepsilon}{3(1+\varepsilon)}<\frac{\varepsilon}{3}
\end{aligned}
$$

and thus

$\left\|P_{1 q}^{*}\left(\varphi(v)-y^{*}\right)\right\|=\sup \left\{\left|\left\langle z, P_{1 q}^{*}\left(\varphi(v)-y^{*}\right)\right\rangle\right|: z \in B\left(\left|y_{m n}\right|\right)\right\} \leq \frac{E}{3}$.

On the other hand, if $I$ denotes the identity map in $\left[y_{m n}\right]$, we have that, for $z$ in $B\left(\left|y_{m n}\right|\right)$ and after (5),

$$
\begin{gathered}
\left|\left\langle z,\left(I^{*}-P_{1 q}^{*}\right) \varphi(v)\right\rangle\right|=\left|\left\langle z, \sum\left\{\left\langle y_{m n}, \varphi(v)\right\rangle y_{m n}^{*}: 1 q<^{*} m n\right\}\right\rangle\right| \\
\leq \sum\left\{\left|\left\langle y_{m n} ; v\right\rangle\right| \cdot\left\|y_{m n}^{*}\right\|: 1 q<^{*} m n\right\} \leq \frac{1}{8} 2 b \sum\left\{\varepsilon_{m n}: 1 q<^{*} m n\right\},
\end{gathered}
$$
and hence $\left.\left\|\left(I^{*}-P_{1 q}^{*}\right) \varphi(v)\right\|=\sup \left\{\left|\left\langle z,\left(I^{*}-P_{1 q}^{*}\right) \varphi(v)\right\rangle\right|: z \in B\left(\mid y_{m n}\right]\right)\right\} \leq \frac{\varepsilon}{3}$. 
Then

$$
\begin{gathered}
\left\|\varphi(v)-y^{*}\right\| \leq\left\|\varphi(v)-P_{1 q}^{*} y^{*}\right\|+\left\|P_{1 q}^{*} y^{*}-y^{*}\right\| \\
\leq\left\|P_{1 q}^{*}\left(\varphi(v)-y^{*}\right)+\left(I^{*}-P_{1 q}^{*}\right) \varphi(v)\right\|+\left\|P_{1 q}^{*} y^{*}-y^{*}\right\| \\
\leq\left\|P_{1 q}^{*}\left(\varphi(v)-y^{*}\right)\right\|+\left\|\left(I^{*}-P_{1 q}^{*}\right) \varphi(v)\right\|+\left\|P_{1 q}^{*} y^{*}-y^{*}\right\|<\varepsilon,
\end{gathered}
$$

whence we deduce that

$$
\overline{\varphi\left(B\left(\left[u_{2 n}\right]\right)\right)}=\overline{\varphi\left(B\left(\left[u_{n}\right]\right)\right)}=B\left(\left[y_{m n}^{*}\right]\right) .
$$

Finally, we make use of the open mapping theorem to obtain that

$$
\varphi:\left\{u_{2 n}\right] \longrightarrow\left[y_{m n}^{*}\right] \text { and } \varphi:\left[u_{n}\right] \longrightarrow\left[y_{m n}^{*}\right]
$$

are topological homomorphisms and

$$
\left.\varphi\left(\stackrel{\circ}{B}\left(\left[u_{2 n}\right]\right)\right)=\varphi\left(\stackrel{\circ}{B}\left[u_{n}\right]\right)\right)=\stackrel{\circ}{B}\left(\left[y_{m n}^{*}\right]\right) .
$$

Proposition 1. Let $X$ be a Banach space. Let $Y$ be a separable norming closed subspace of $X^{*}$. Let $\left(x_{m n}\right)$ be a double sequence in $X$ such that, for every $m$ in $\mathbb{N},\left(x_{m n}\right)_{n=1}^{\infty}$ has a weak ${ }^{*}$-cluster point $x_{m}$ in $X^{* *}$. If

$$
\text { inf }\left\{\left\|x_{m n}\right\|: n \in N\right\}>0, \quad m \in N,
$$

and

$$
\text { inf }\left\{\left\|x_{m n}-x_{m}\right\|: n \in \mathbb{N}\right\}>0, m \in \mathbb{N},
$$

(notice that the second condition is satisfied automatically when $x_{m} \in$ $\left.X^{* *} \backslash X\right)$ then the following properties are satisfied:

1. There is a subsequence $\left(y_{m n}\right)_{n=1}^{\infty}$ of $\left(x_{m n}\right)_{n=1}^{\infty}, m \in \mathbb{N}$, such that $y_{11}-x_{1}, y_{12}-x_{1}, y_{21}-x_{2}, \cdots, y_{1 n}-x_{1}, y_{2(n-1)}-x_{2}, \cdots, y_{(n-1) 2}-x_{n-1}, \cdots$, $y_{n 1}-x_{n}, \ldots$

is a basic sequence in $X^{* *}$ which is also a subsequence of $x_{11}-x_{1}, x_{12}-x_{1}, x_{21}-x_{2}, \cdots, x_{1 n}-x_{1}, x_{2(n-1)}-x_{2}, \cdots, x_{(n-1) 2}-x_{n-1}, \cdots$, $x_{n 1}-x_{n}, \cdots$ 
2. If the subspace of $X^{*}$ orthogonal to $\left[x_{m}\right]$ contains $Y$, then $\left(y_{m n}\right)$ may be chosen in such a way that

$$
y_{11}, y_{12}, y_{21}, \ldots, y_{1 n}, y_{2(n-1)}, \ldots, y_{(n-1) 2}, \ldots, y_{n 1}, \ldots
$$

be a basic sequence in $X$ and, if $\varphi$ is the canonical mapping from $X^{*}$ onto $X^{*} /\left[y_{m n}\right]^{\perp}$, then $\varphi(Y)=\left[y_{m n}\right]^{*}$.

Proof. We may assume, conveniently renorming $X$ if necessary, that $Y$ is 1-norming. In $X^{* *}$, let $Z$ be the closed linear span of $\left\{x_{m n}: m, n \in\right.$ $\mathbb{N}\} \cup\left\{x_{m}: m \in \mathbb{N}\right\}$. We take a countable dense subset $\left\{z_{n}: n \in \mathbb{N}\right\}$ in $Z$. For each pair of positive integers $m, n$, we find an element $u_{m n}$ in $X^{*}$ such that

$$
\left\|u_{m n}\right\|=1, \quad\left|\left\langle z_{n}, u_{m n}\right\rangle\right|>\left\|z_{n}\right\|-\frac{1}{m} .
$$

Clearly, $\left[u_{m n}\right]$ is a 1-norming subspace of $X^{* * *}$ with respect to $Z$. Let $\left\{v_{p}\right\}$ be a countable dense subset of $Y$. For each positive integer $m$, we find a subsequence $\left(z_{m n}\right)_{n=1}^{\infty}$ of $\left(x_{m n}\right)_{n=1}^{\infty}$ such that

$$
\lim _{n}\left\langle z_{m n}-x_{m}, u\right\rangle=0, \quad m \in \mathbb{N}, \quad u \in\left\{u_{m n}\right\} \cup\left\{v_{p}\right\},
$$

and

$$
z_{11}, z_{12}, z_{21}, \ldots, z_{1 n}, z_{2(n-1)}, \ldots, z_{(n-1) 2}, z_{n 1}, \ldots
$$

is a subsequence of

$$
x_{11}, x_{12}, x_{21}, \ldots, x_{1 n}, x_{2(n-1)}, \ldots, x_{(n-1) 2}, x_{n 1}, \ldots
$$

We now apply Lemma 2 to obtain a subsequence $\left(y_{m n}\right)_{n=1}^{\infty}$ of $\left(z_{m n}\right)_{n=1}^{\infty}$, $m \in \mathbb{N}$, such that

$$
\begin{gathered}
y_{11}-x_{1}, y_{12}-x_{1}, y_{21}-x_{2}, \cdots, y_{1 n}-x_{1}, y_{2(n-1)}-x_{2}, \cdots, y_{(n-1) 2}-x_{n-1}, \cdots, \\
y_{n 1}-x_{n}, \cdots
\end{gathered}
$$

be a basic sequence of $X^{* *}$ such that it is a subsequence of $z_{11}-x_{1}, z_{12}-x_{1}, z_{21}-x_{2}, \cdots, z_{1 n}-x_{1}, z_{2(n-1)}-x_{2}, \cdots, z_{(n-1) 2}-x_{n-1}, \cdots$, $z_{n 1}-x_{n}, \cdots$ 
It is clear that $\lim _{n}\left\langle y_{m n}-x_{m}, u\right\rangle=0, m \in N$ and $u \in\left\{v_{p}\right\}$, thus, if the subspace of $X^{*}$ orthogonal to $\left[x_{m}\right]$ contains $Y$, it follows that

$$
\lim _{n}\left\langle y_{m n}, u\right\rangle=0, \quad m \in \mathbb{N}, u \in\left\{v_{p}\right\}
$$

and since $Y=\left[v_{p}\right]$ is separable and 1-norming, we may choose $\left(y_{m n}\right)_{m=1}^{\infty}$, $m \in \mathbb{N}$, after Lemma 2, so that

$$
y_{11}, y_{12}, y_{21}, \ldots, y_{1 n}, y_{2(n-1)}, \ldots, y_{(n-1) 2}, y_{n 1}, \ldots
$$

is a basic sequence in $X$ such that, if $\varphi$ is the canonical mapping from $X^{*}$ onto $X^{*} /\left[y_{m n}\right]^{\perp}$, then $\varphi(Y)$ coincides with $\left[y_{m n}^{*}\right]$.

Theorem 1. Let $X$ be a Banach space with $X^{*}$ separable. Let $Y$ be a norming closed subspace of $X^{*}$. Then there is a basic sequence $\left(z_{n}\right)$ in $X$ such that $X^{*} / Y$ is isometric to $\left[z_{n}\right]^{*} /\left[z_{n}^{*}\right]$.

Proof. Since $B\left(X^{* *}\right)$ is compact and metrizable for the weak* topology, we find in $B\left(X^{* *}\right) \cap Y^{\perp}$ a countable subset $\left\{x_{1}, x_{2}, \ldots, x_{m}, \ldots\right\}$ that is weak $^{*}$ dense. For each positive integer $m$, we choose a sequence $\left(x_{m n}\right)_{n=1}^{\infty}$ in $X$ that $\sigma\left(X^{* *}, X^{*}\right)$-converges to $x_{m}$ in such a way that

$\inf \left\{\left\|x_{m n}\right\|: n \in \mathbb{N}\right\}>0, \quad$ inf $\left\{\left\|x_{m n}-x_{m}\right\|: n \in \mathbb{N}\right\}>0, m \in N$.

This selection is guaranteed by the fact that weak*-neighborhoods are always infinite-dimensional and also that the closed ball centered at zero with radius $\left\|x_{m}\right\| / 2$ is weak ${ }^{*}$-closed in $X^{* *}$. We then apply the former proposition to obtain a subsequence $\left(y_{m n}\right)_{\pi=1}^{\infty}$ of $\left(x_{m n}\right)_{n=1}^{\infty}, m \in \mathbb{N}$, such that

$$
y_{11}, y_{12}, y_{21}, \ldots, y_{1 n}, y_{2(n-1)}, \ldots, y_{(n-1) 2}, y_{n 1}, \ldots
$$

be a basic sequence in $X$ so that, if $\varphi$ denotes the canonical mapping from $X^{*}$ onto $X^{*} /\left[y_{m n}\right]^{\perp}$, then $\varphi(Y)=\left[y_{m n}^{*}\right]$. We write such a sequence as $\left(z_{n}\right)$. It then follows that $\left[z_{n}\right]^{\perp}$ is contained in $Y$, from where we easily deduce that $X^{*} / Y$ is isometric to $\left[z_{n}\right]^{*} /\left[z_{n}^{*}\right]$.

Proposition 2. Let $X$ be a Banach space. Let $V$ be a 1-norming closed subspace of $X^{*}$. If $W$ is a separable norming subspace of $X^{*}$ with $W \cap V=\{0\}$, then there is a closed separable subspace $Y$ of $X$ such that in $Y^{*}$ there are a 1-norming closed subspace $V_{1}$ and a norming subspace $W_{1}$ with $V_{1} \cap W_{1}=\{0\}$ and $W_{1}$ is isometric to $W$. 
Proof. Let $r$ be a positive integer. For every $x$ in $X$, we choose an element $u(x, r)$ in $V$ such that

$$
\|u(x, r)\|=1, \quad|\langle x, u(x, r)\rangle|>\|u\|-\frac{1}{r} .
$$

We take in $X$ a countable subset $A_{0}$ and in $W$ a countable dense subset $B_{0}$. We proceed by recurrence and suppose that, for a non-negative integer $n$, we have obtained countable subsets $A_{n}$ and $B_{n}$ in $X$ and $X^{*}$, respectively. Let $C_{n}$ and $D_{n}$ denote the linear spans over $H$ of $A_{n}$ and $B_{n}$, respectively. We define

$$
\begin{aligned}
& A_{n+1}:=C_{n} \cup\left\{x(u, r): u \in D_{n}, r \in N\right\}, \\
& B_{n+1}:=D_{n} \cup\left\{u(x, r): x \in C_{n}, r \in \mathbb{N}\right\} .
\end{aligned}
$$

We put $Y$ and $F$ to denote the closures in $X$ and $X^{*}$ of $\cup_{n=0}^{\infty} A_{n}$ and $\cup_{n=0}^{\infty} B_{n}$, respectively. Clearly, we have that $Y$ and $F$ are Banach spaces. Given $u \in F, v \in Y^{\perp}$ and $\varepsilon>0$, we find two positive integers $n, r$ and $w$ in $B_{n}$ such that $\frac{1}{\tau}<\varepsilon$ and $\|u-w\|<\varepsilon$. Then

$$
\begin{gathered}
\|u\| \leq\|u-w\|+\|w\|<\varepsilon+|\langle x(w, r), w\rangle|+\frac{1}{r} \\
\leq 2 \varepsilon+|\langle x(w, r), w+v\rangle| \leq 2 \varepsilon+|\langle x(w, r), w-u\rangle| \\
+|\langle x(w, r), u+v\rangle| \\
\leq 2 \varepsilon+\|w-u\|+\|u+v\| \leq 3 \varepsilon+\|u+v\|,
\end{gathered}
$$

and thus

$$
\|u\| \leq\|u+v\|
$$

from where we deduce that $Y^{\perp}$ is an orthogonal complement of $F$ in $F+Y^{\perp}$. We then have that $W$ is contained in $F$ and if $\psi$ is the canonical mapping from $X^{*}$ onto $X^{*} / Y^{\perp}, \psi: F \longrightarrow X^{*} / Y^{\perp}=Y^{*}$ is an into isometry. We put $W_{1}:=\psi(W)$ and $V_{1}:=\psi(F \cap V)$. It clearly now follows that $W_{1}$ is isometric to $W$ and $V_{1} \cap W_{1}=\{0\}$. On the other hand, given $x$ in $Y$ and $\varepsilon>0$, we find $m, r \in \mathbb{N}$ and $z$ in $A_{m}$ so that $\|x-z\|<\varepsilon, \frac{1}{r}<\varepsilon$. Then,

$$
\psi(u(z, r)) \in V_{1}, \quad\|\psi(u(z, r))\|=1
$$


and

$$
\begin{aligned}
& |\langle x, \psi(u(z, r))\rangle|=|\langle x, u(z, r)\rangle| \geq|\langle z, u(z, r)\rangle|-|\langle x-z, u(z, r)\rangle| \\
& \geq\|z\|-\frac{1}{r}-\|x-z\| \geq\|x\|-\frac{1}{r}-2\|x-z\| \geq\|x\|-3 \varepsilon,
\end{aligned}
$$

therefore we deduce that $V_{1}$ is 1-norming.

In order to prove Lemma 3 we shall need the following result of Kadec and Klee's, [11] and [12] (see also [2]): b) Let $X$ be a separable Banach space and let $Y$ be a separable closed subspace of $X^{*}$. Then there is an equivalent norm $\|\cdot\|$ in $X$ such that, if $\left(x_{n}^{*}\right)$ is an arbitrary sequence in $X^{*}$ that converges to an element $x^{*}$ of $Y$ respect to the weak topology and such that $\left(\left\|x_{n}^{*}\right\|\right)$ converges to $\left\|x^{*}\right\|$, then the sequence $\left(\left\|\mid x_{n}^{*}-x^{*}\right\|\right)$ converges to zero.

Lemma 3. Let $X$ be a separable Banach space. Let $Y$ be a closed separable subspace of $X^{*}$. Let $\left(u_{m n}\right)$ be a double sequence in $X^{*}$ such that, for each $m$ in $N$, the sequence $\left(u_{m n}\right)_{n=1}^{\infty}$ converges to the origin for the weak ${ }^{*}$ topology and

$$
\text { inf }\left\{\left\|u_{m n}\right\|: n \in N\right\}>0 \text {. }
$$

If $\left(u_{m n}\right)$ is in $Y$, then there is a subsequence $\left(v_{m n}\right)_{n=1}^{\infty}$ of $\left(u_{m n}\right)_{n=1}^{\infty}$, $m \in \mathbb{N}$, satisfying the following conditions:

1.

$$
v_{11}, v_{12}, v_{21}, \ldots, v_{1 n}, v_{2(n-1)}, \ldots, v_{(n-1) 2}, v_{n 1}, \ldots
$$

is a $w^{*}$-basic sequence in $X^{*}$ which is a subsequence of

$$
u_{11}, u_{12}, u_{21}, \ldots, u_{1 n}, u_{2(n-1)}, \ldots, u_{(n-1) 2}, u_{n 1}, \ldots
$$

so that if $\left(w_{n}\right)$ is an arbitrary subsequence of $(10)$, then $\left[w_{n}\right]$ is $\sigma\left(X^{*}, X\right)$-closed in $Y$.

2. $\left[v_{m n}\right\}$ is a 1 -norming subspace of $\left(X /\left[v_{m n}\right]_{\perp}\right)^{*}=\left[v_{m n}\right]$.

Proof. We apply b) to obtain an equivalent norm $\|\cdot\|$ in $X$ with the property there mentioned. We write $Z:=(X,\|\cdot\|)$. Let $\left\{x_{1}, x_{2}, \ldots, x_{n}, \ldots\right\}$ be a dense subset of $Z$. Then

$$
\lim _{n}\left\langle x_{j}, u_{m n}\right\rangle=0, \quad m, n \in \mathbb{N},
$$


and since $Z$ is a 1-norming subspace of $Z^{* *}$, we apply Lemma 2 replacing $X$ by $Z^{*}, X^{*}$ by $Z^{* *}, u_{2 n-1}$ by $x_{n}, u_{2 n}$ by $x_{n}, n \in N$, and $u_{m n}$ by $x_{m n}$, and thus we obtain a subsequence $\left(w_{m n}\right)_{n=1}^{\infty}$ of $\left(u_{m n}\right)_{n=1}^{\infty}, m \in \mathbb{N}$, such that

$$
w_{11}, w_{12}, w_{21}, \ldots, w_{1 n}, w_{2(n-1)}, \ldots, w_{(n-1) 2}, w_{n 1}, \ldots
$$

is a basic sequence in $X^{*}$ which is a subsequence of

$$
u_{11}, u_{12}, u_{21}, \ldots, u_{1 n}, u_{2(n-1)}, \ldots, u_{(n-1) 2}, u_{n 1}, \ldots
$$

so that, if $\varphi$ is the canonical mapping from $Z^{* *}$ onto $Z^{* *} /\left[w_{m n}\right]^{\perp}$, then $\left.\varphi(\stackrel{B}{B}(Z))=\stackrel{\circ}{B}\left(\mid w_{m n}^{*}\right]\right)$. Let $\psi$ denote the canonical mapping from $Z$ onto $Z /\left[w_{m n}\right]_{\perp}$. Given an element $z$ in $Z /\left[w_{m n}\right]_{\perp}$, we find $z_{1}$ in $Z$ such that $\psi\left(z_{1}\right)=z$ and write $\Phi(z):=\varphi\left(z_{1}\right)$. Then, $\Phi$ is a one-to-one linear map from $Z /\left[w_{m n}\right]_{\perp}$ onto $\left[w_{m n}^{*}\right]$. For a given $\varepsilon>0$, we may choose the above mentioned $z_{1}$ in such a way that $\left\|z_{1}\right\|<\|z\|+\varepsilon$ and hence

$$
\|\Phi(z)\|=\left\|\varphi\left(z_{1}\right)\right\| \leq\left\|z_{1}\right\|<\|z\|+\varepsilon
$$

and, consequently, $\|\Phi\| \leq 1$. On the other hand, given $y$ in $\left[w_{m n}^{*}\right]$ and $\varepsilon>0$, we deduce from $\varphi(\stackrel{\circ}{B}(Z))=\stackrel{\circ}{B}\left(\left[w_{m+n}^{*}\right]\right)$ that there is a $y_{1}$ in $Z$ such that

$$
\varphi\left(y_{1}\right)=y, \quad\left\|y_{1}\right\|<\|y\|+\varepsilon
$$

and so

$$
\left\|\Phi^{-1}(y)\right\|=\left\|\psi\left(y_{1}\right)\right\| \leq\left\|y_{1}\right\|<\|y\|+\varepsilon
$$

and, consequently, $\left\|\Phi^{-1}\right\| \leq 1$. Hence we have shown that

$$
\Phi: Z /\left[w_{m n}\right]_{\perp} \longrightarrow\left[w_{m n}^{*} \mid\right.
$$

is an isometry. If we now write $z_{m n}: \equiv \Phi^{-1}\left(w_{m n}^{*}\right)$, it follows that

$$
z_{11}, z_{12}, z_{21}, \ldots, z_{1 n}, z_{2(n-1)}, \ldots, z_{(n-1) 2}, z_{n 1}, \ldots
$$

is a Schauder basis in $Z /\left[w_{m \pi}\right]_{\perp}$ whose associated linear functionals are (11). Consequently, (11) is a $\mathrm{w}^{*}$-basic sequence in $X^{*}$. Given now $z$ in $Z /\left[w_{m n}\right]_{\perp}$, we find $z_{1}$ in $Z$ such that $\psi\left(z_{1}\right)=z$. Then, if $w$ belongs to $\left[w_{m n}\right]$, we have that

$$
|\langle z, w\rangle|=\left|\left\langle z_{1}, w\right\rangle\right|=\left|\left\langle\varphi\left(z_{1}\right), w\right\rangle\right|=|\langle\Phi(z), w\rangle|,
$$


whence we conclude that

$$
\begin{gathered}
\|z\|=\|\Phi(z)\|=\sup \left\{|\langle\Phi(z), w\rangle|: w \in B\left(\left|w_{m n}\right|\right)\right\} \\
=\sup \left\{|\langle z, w\rangle|: w \in B\left(\left[w_{m n} \mid\right)\right\}\right.
\end{gathered}
$$

and hence, $\left[w_{m n}\right]$ is a 1 -norming subspace of $\left(Z /\left[w_{m n}\right]_{\perp}\right)^{*}=\left[w_{m n}\right]$. We take now an element $v$ in $Y \cap\left[w_{m n}\right] \sim\|v\|=1$. We may find a sequence $\left(v_{n}\right)$ in $\left|w_{m n}\right|,\left\|v_{n}\right\|=1, n \in \mathbb{N}$, such that it converges to $v$ for the weak* topology. Then, after result b), $\left(v_{n}\right)$ converges to $v$ in norm, from where we have that $v$ is in $\left[w_{m n}\right]$ and thus this space is $\sigma\left(X^{*}, X\right)$-closed in $Y$.

Let $\left(w_{n}\right)$ be a subsequence of (11). We write the sequence (11) in the form $\left(y_{n}\right)$ and the subsequence $\left(w_{n}\right)$ as $\left(y_{n_{j}}\right)$. Let $w$ be an element of $\left[w_{n}\right]^{\sim} \cap Y$. Then for the weak* topology,

$$
w=\sum_{j=1}^{\infty} a_{j} y_{n_{j}},
$$

which also allows us to write, again with respect to the weak* topology,

$$
w=\sum_{n=1}^{\infty} b_{n} y_{n},
$$

with $b_{n}=0$ for those $n$ different from the $n_{j}$. We then have that $w$ is an element of $\left[w_{n}\right] \sim \cap Y$ and, after what has already been shown, $w \in\left[w_{m n}\right]$, from where we deduce that $w=\sum_{j=1}^{\infty} a_{j} y_{n_{j}} \in\left[w_{n}\right]$, and so we conclude that $\left[w_{n}\right]$ is $\sigma\left(X^{*}, X\right)$-closed in $Y$.

Theorem 2. Let $X$ be a separable Banach space. Let $V$ be a norming closed subspace of $X^{*}$. Let $\left(x_{m n}\right)$ be a double sequence in $X$ such that, for each $m \in \mathbb{N}$,

$$
\lim _{n}\left\langle x_{m n}, v\right\rangle=0, \quad v \in V, \quad \text { inf }\left\{\left\|x_{m n}\right\|: n \in \mathbb{N}\right\}>0 .
$$

Then, there is a subsequence $\left(y_{m n}\right)_{n=1}^{\infty}$ of $\left(x_{m n}\right)_{n=1}^{\infty}, m \in \mathbb{N}$, such that

$$
y_{11}, y_{12}, y_{21}, \ldots, y_{1 n}, y_{2(n-1)}, \ldots, y_{(n-1) 2}, y_{n 1}, \ldots
$$

is a basic sequence in $X$ which is a subsequence of

$$
x_{11}, x_{12}, x_{21}, \ldots, x_{1 n}, x_{2(n-1)}, \ldots, x_{(n-1) 2}, x_{n 1}, \ldots
$$


so that, if $\left(z_{n}\right)$ is an arbitrary subsequence of $(13)$, then $\left[z_{n}\right]$ is $\sigma(X, V)$ closed in $X$.

Proof. Clearly, we may assume that $V$ is separable. If $\varphi$ is the canonical mapping from $X^{* *}$ onto $X^{* *} / V^{\perp}$, then we have, since $V$ is norming, that $\varphi(X)$ is a subspace of $V^{*}=X^{* *} / V^{\perp}$ isomorphic to $X$. Thus $\varphi(X)$ is a separable closed subspace of $V^{*}$ and $\left(\varphi\left(x_{m n}\right)\right)$ is a double sequence in $\varphi(X)$ such that, for each $m$ of $N,\left(\varphi\left(x_{m n}\right)\right)_{n=1}^{\infty}$ converges to the origin for the weak ${ }^{*}$ topology and

$$
\inf \left\{\left\|\varphi\left(x_{m n}\right)\right\|: n \in \mathbb{N}\right\}>0 .
$$

We apply Lemma 3 and obtain a subsequence $\left(y_{m n}\right)_{n=1}^{\infty}$ of $\left(x_{m n}\right)_{n=1}^{\infty}$, $m \in \mathbb{N}$, such that

$$
\varphi\left(y_{11}\right), \varphi\left(y_{12}\right), \varphi\left(y_{21}\right), \ldots, \varphi\left(y_{1 n}\right), \varphi\left(y_{2(n-1)}\right), \ldots, \varphi\left(y_{(n-1) 2}\right), \varphi\left(y_{n 1}\right), \ldots
$$

is a $\mathrm{w}^{*}$-basic sequence in $V^{*}$ which is a subsequence of

$$
\varphi\left(x_{11}\right), \varphi\left(x_{12}\right), \varphi\left(x_{21}\right), \ldots, \varphi\left(x_{1 n}\right), \varphi\left(x_{2(n-1)}\right), \ldots, \varphi\left(x_{(n-1) 2}\right), \varphi\left(x_{n 1}\right), \ldots
$$

so that, if $\left(w_{n}\right)$ is an arbitrary subsequence of $(13)$, then $\left[w_{n}\right]$ is $\sigma\left(V^{*}, V\right)$ closed in $\varphi(X)$. It is now immediate that

$$
y_{11}, y_{12}, y_{21}, \ldots, y_{1 n}, y_{2(n-1)}, \ldots, y_{(n-1) 2}, y_{n 1}, \ldots
$$

satisfying the statement of the theorem.

Theorem 3. Let $X$ be a separable Banach space. Let $U \subset V$ separable norming closed subspaces of $X^{*}$. Let $Z$ be a normed separable subspace of $X^{*}$ such that $Z \cap V=\{0\}$. Then there is a double sequence $\left(x_{m n}\right)$ in $X$ such that

$$
x_{11}, x_{12}, x_{21}, \ldots, x_{1 n}, x_{2(n-1)}, \ldots, x_{(n-1) 2}, x_{n 1}, \ldots
$$

is a basic sequence in $X$ and if $\left(z_{m n}\right)_{n=1}^{\infty}$ is an arbitrary subsequence of - $\left(x_{m n}\right)_{n=1}^{\infty}, m \in N$, then there is a subsequence $\left(y_{m n}\right)_{n=1}^{\infty}$ of $\left(z_{m n}\right)_{n=1}^{\infty}$, $m \in \mathbb{N}$, such that 
is a subsequence of (14) and if $\varphi$ is the canonical mapping from $X^{*}$ onto $X^{*} /\left|y_{m n}\right|^{\perp}$, we have that

$$
\begin{gathered}
\left(U \cap\left[y_{m n}\right]^{\perp}\right)^{\sim}=\left[y_{m n}\right]^{\perp}, \varphi(U)=\varphi(V)=\left[y_{m n}^{*}\right], \\
V \subset U+\left[y_{m n}\right]^{\perp}, \quad\left(V+\left[y_{m n}\right]^{\perp}\right) \cap Z=\{0\} .
\end{gathered}
$$

Proof. We may assume, by conveniently renorming $X$ if necessary, that $U$ is 1-norming. Let $S\left(X^{* *}\right)=\left\{x^{* *} \in X^{* *}:\left\|x^{* *}\right\|=1\right\}$. Since $Z \cap V=\{0\}$ and $Z$ being separable, we may find in $V^{\perp} \cap S\left(X^{* *}\right)$ a sequence $\left(t_{n}\right)$ so that $\left\{t_{m}: m \in \mathbb{N}\right\}$ separates the elements of $Z$. Now, since $V$ and $Z$ are separable, we may find in $X$ a sequence $\left(s_{m n}\right)_{n=1}^{\infty}$ converging to $t_{m}$ in every point of $V+Z$ and such that, for each $m$,

$$
\text { inf }\left\{\left\|s_{m n}\right\|: n \in \mathbb{N}\right\}>0 \text {. }
$$

We apply Theorem 2 to obtain a subsequence $\left(x_{m n}\right)_{n=1}^{\infty}$ of $\left(s_{m n}\right)_{n=1}^{\infty}$, $m \in \mathbb{N}$, so that

$$
x_{11}, x_{12}, x_{21}, \ldots, x_{1 n}, x_{2(n-1)}, \ldots, x_{(n-1) 2}, x_{n 1}, \ldots
$$

be a basic sequence in $X$ and if $\left(x_{n}\right)$ is an arbitrary subsequence of (15), $\left[x_{n}\right]$ is $\sigma(X, U)$-closed in $X$. We take an arbitrary subsequence $\left(z_{m n}\right)_{n=1}^{\infty}$ of $\left(x_{m n}\right)_{n=1}^{\infty}, m \in \mathbb{N}$. From $\left(z_{m n}\right)_{n=1}^{\infty}$ we extract a subsequence, which we shall still denote by $\left(z_{m n}\right)_{n=1}^{\infty}$, such that

$$
z_{11}, z_{12}, z_{21}, \ldots, z_{1 n}, z_{2(n-1)}, \ldots, z_{(n-1) 2}, z_{n 1}, \ldots
$$

is a subsequence of (15). Now, since $U$ is 1-norming, $\lim _{n}\left\langle z_{m n}, v\right\rangle=0$, $m \in N, v \in V$, and inf $\left\{\left\|z_{m n}\right\|: n \in \mathbb{N}\right\}>0$, we obtain, applying Lemma 2, a subsequence $\left(y_{m n}\right)_{n=1}^{\infty}$ of $\left(z_{m n}\right)_{n=1}^{\infty}, m \in N$, such that

$$
y_{11}, y_{12}, y_{21}, \ldots, y_{1 n}, y_{2(n-1)}, \ldots, y_{(n-1) 2}, y_{n 1}, \ldots
$$

is a subsequence of (16) and if $\varphi$ is the canonical mapping from $X^{*}$ onto $X^{*} /\left[y_{m n}\right]^{\perp}$, then

$$
\varphi(\stackrel{\circ}{B}(U))=\varphi(\stackrel{\circ}{B}(V))=\stackrel{\circ}{B}\left(\left[y_{m n}^{*}\right]\right) .
$$

Now, since $\left[y_{m n}\right]$ is $\sigma(X, U)$-closed in $X$, one has that, after the bipolar theorem,

$$
\left(U \cap\left[y_{m n}\right]^{\perp}\right)^{\sim}=\left(U^{\perp}+\left(\left[y_{m n}\right]^{\perp}\right)_{\perp}\right)^{\perp}=\left[y_{m n}\right]^{\perp} .
$$


We deduce from (17) that $\varphi(U)=\varphi(V)=\left[y_{m n}^{*}\right]$. If $v$ is an arbitrary element of $V$ we may find $u$ in $U$ such that $\varphi(u)=\varphi(v)$. Then, $v \in$ $u+\left[y_{m n}\right]^{\perp}$ and, therefore

$$
V \subset U+\left[y_{m n}\right]^{\perp}
$$

Finally, if there is an element $w$ in $Z$, different from the origin, then there is a positive integer $m$ such that $\left\langle t_{m}, v\right\rangle \neq 0$. Let $v$ be any element in $V$. Then

$$
0 \neq\left\langle t_{m}, w\right\rangle=\left\langle t_{m}, w-v\right\rangle=\lim _{n}\left\langle y_{m n}, w-v\right\rangle
$$

and hence $w-v$ is not in $\left[y_{m n}\right]^{\perp}$. Consequently,

$$
\left(V+\left[y_{m n}\right]^{\perp}\right) \cap Z=\{0\} .
$$

Corollary 1. Let $X$ be a Banach space with $X^{*}$ separable. Let $U \subset V$ be two norming closed subspaces of $X^{*}$. Then there is a basic sequence $\left(x_{n}\right)$ in $X$ such that

$$
\left(U \cap\left[x_{n}\right]^{\perp}\right)=\left[x_{n}\right]^{\perp} \text { and } U+\left[x_{n}\right]^{\perp}=V .
$$

Proof. We can take a separable normed subspace $Z$ of $X^{*}$ such that $Z \cap V=\{0\}$ and $Z+V=X^{*}$. We apply the former theorem and so obtain a double sequence $\left(y_{m n}\right)$ in $X$ with the properties there stated. We write the sequence

$$
y_{11}, y_{12}, y_{21}, \ldots, y_{1 n}, y_{2(n-1)}, \ldots, y_{(n-1) 2}, y_{n 1}, \ldots
$$

in the form $\left(x_{n}\right)$. It is then immediate that

$$
\left(U \cap\left[x_{n}\right]^{\perp}\right)^{-}=\left[x_{n}\right]^{\perp} \text { and } U+\left[x_{n}\right]^{\perp}=V .
$$

Corollary 2. Let $X$ be a Banach space with $X^{* *}$ separable. Let $Y$ be a closed subspace of $X^{* *}$ with $X \subset Y$. Then there is a $w^{*}$-basic sequence $\left(x_{n}\right)$ in $X^{*}$ such that

$$
\left.\left(\mid x_{n}\right]_{\perp}\right)^{-}=\left[x_{n}\right]^{\perp}, \quad X+\left|x_{n}\right|^{\perp}=Y,
$$


and $\left[x_{n}\right]_{\perp}$ has infinite dimension.

Proof. We take a normed separable subspace $Z$ in $X^{* *}$ such that $Z \cap Y=\{0\}$ and $Z+Y=X^{* *}$. We apply Theorem 3 switching $X$ by $X^{*}, U$ by $X$ and $V$ by $Y$ and obtain a double sequence $\left(x_{m n}\right)$ in $X^{*}$ with the properties there stated. Then, by applying Lemma 3 to the double sequence $\left(x_{m n}\right)$ in the separable space $X^{*}$ we obtain a subsequence $\left(z_{m n}\right)_{n=1}^{\infty}$ of $\left(x_{m n}\right)_{n=1}^{\infty}, m \in \mathbb{N}$, in such a way that

$$
z_{11}, z_{12}, z_{21}, \ldots, z_{1 n}, z_{2(n-1)}, \ldots, z_{(n-1) 2}, z_{n 1}, \ldots
$$

be a $w^{*}$-basic subsequence of $x_{11}, x_{12}, x_{21}, \ldots$, It is now evident that $z_{11}, z_{12}, z_{21}, \ldots, z_{1 n}, z_{2(n-1)}, \ldots, z_{(n-1) 2}, z_{n 1}, \ldots$ may be chosen so that $\left[z_{m n}\right]_{\perp}$ has infinite dimension, by considering, if necessary, convenient subsequences of $\left(z_{m n}\right)_{n=1}^{\infty}, m \in \mathbb{N}$.

Now, Theorem 3 guarantees that there is a subsequence $\left(y_{m n}\right)_{n=1}^{\infty}$ of $\left(z_{m n}\right)_{n=1}^{\infty}, m \in \mathbb{N}$, such that

$$
y_{11}, y_{12}, y_{21}, \ldots, y_{1 n}, y_{2(n-1)}, \ldots, y_{(n-1) 2}, y_{n 1}, \ldots
$$

is a subsequence of $z_{11}, z_{12}, z_{21}, \ldots, z_{1 n}, z_{2(n-1)}, \ldots, z_{(n-1) 2}, z_{n 1}, \ldots$ and satisfies the properties stated in Theorem 3 and also those of Corollary 1 , replacing $X$ and $X^{*}$ by $X^{*}$ and $X^{* *}$, and $U$ and $V$ by $X$ and $Y$. Hence,

$$
\left(X \cap\left[y_{m n}\right]^{\perp}\right)^{\sim}=\left[y_{m n}\right]^{\perp}, \quad X+\left[y_{m n}\right]^{\perp}=Y .
$$

If we now write the sequence $y_{11}, y_{12}, y_{21}, \ldots, y_{1 n}, y_{2(n-1)}, \ldots, y_{(n-1) 2}, y_{n 1}, \ldots$ in the form $\left(x_{n}\right)_{n=1}^{\infty}$, we have a $w^{*}$-basic sequence which satisfies

$$
\left(\left[x_{n}\right]_{\perp}\right)^{\sim}=\left(X \cap\left[x_{n}\right]^{\perp}\right)^{\sim}=\left[x_{n}\right]^{\perp}, \quad X+\left[x_{n}\right]^{\perp}=Y .
$$

Finally, from $\left[z_{n}\right]_{\perp} \subset\left[x_{n}\right]_{\perp}$ it follows that $\left[x_{n}\right]_{\perp}$ has infinite dimension, which concludes the proof.

If in the previous corollary we take $Y=X$, it follows that $\left[x_{n}\right]_{\perp}$ is a subspace of $X$ which is reflexive and of infinite dimension. Consequently, $X$ and $X^{*}$ are somewhat reflexive, [10]: On the other hand, let us suppose that $k$ is a positive integer less or equal than the dimension of $X^{* *} / X$. We may take $Y$ so that $Y / X$ has dimension $k$ and thus $\left[x_{n}\right]_{\perp}$ is quasi-reflexive of order $k$; if we take $Y$ in such a way that $X^{* *} / Y$ 
has dimension $k$, then $X /\left|x_{n}\right|_{\perp}$ is quasi-reflexive of order $k$. As a consequence, we have that in $X$ and $X^{*}$ there are quasi-reflexive subspaces of order $k,[18]$. Corollary 2 also implies that quasi-reflexive subspaces of order $k$ are "twisted sums" of quasi-reflexive spaces of order 1 . Actually, it is an open question to know if there exists a quasi-reflexive space of order 2 which is not a direct sum of two quasi-reflexive spaces of order 1.

Corollary 3. Let $X$ be a Banach space, clearly non quasi-reflexive, such that $X^{*}$ has a norming closed subspace $U$ of infinite codimension. Then there is a basic sequence $\left(x_{n}\right)$ in $X$ such that it has the following properties:

1. $X /\left[x_{n}\right]$ is not quasi-reflexive.

2. $\left[x_{n}\right]$ is not $k$-shrinking for every non-negative integer $k$.

Proof. We may assume, after Proposition 2, that both $X$ and $U$ are separable. It is immediate that we may find in $X^{*}$ a separable closed subspace $V$ containing $U$ and a separable normed subspace $Z$ such that the dimensions of $V / U$ and $Z$ be infinite and $Z \cap V=\{0\}$. We apply now Theorem 3 and obtain a double sequence $\left(y_{m n}\right)$ in $X$ with the properties there stated. We write the sequence

$$
y_{11}, y_{12}, y_{21}, \ldots, y_{1 n}, y_{2(n-1)}, \ldots, y_{(n-1) 2}, y_{n 1}, \ldots
$$

in the form $\left(x_{n}\right)$. From

$$
\left(U \cap\left[x_{n}\right]^{\perp}\right)^{\sim}=\left[x_{n}\right]^{\perp}=\left(X /\left[x_{n}\right]\right)^{*} \text { and } V \subset U+\left[x_{n}\right]^{\perp},
$$

we deduce that $\left(X /\left[x_{n}\right]\right)^{*}$ has a weak ${ }^{*}$-dense subspace $U \cap\left[x_{n}\right]^{\perp}$ of infinite codimension and, hence, $\left.X / \mid x_{n}\right]$ is not quasi-reflexive. From

$$
\left(V+\left[x_{n}\right]^{\perp}\right) \cap Z=\{0\},
$$

we obtain, if $\varphi$ is the canonical mapping from $X^{*}$ onto $X^{*} /\left[x_{n}\right]^{\perp}$, that $\varphi_{\mid Z}$ is one-to-one and, since $\varphi(U)=\varphi(V)=\left[x_{n}^{*}\right]$, it follows that $\varphi(Z) \cap$ $\left[x_{n}^{*}\right]=\{0\}$ and the conclusion follows. 
Corollary 4. Let $X$ be a Banach space with $X^{*}$ separable. If $X$ is not quasi-reflexive, then there is a boundedly complete $w^{*}$-basic sequence $\left(x_{n}\right)$ in $X^{*}$ with the following properties:

1. $\left(\left[x_{n}\right]_{\perp}\right)^{\sim}=\left[x_{n}\right]^{\perp}$ and $\left[x_{n}\right]_{\perp}$ is not quasi-reflexive.

2. $X /\left[x_{n}\right]_{\perp}$ is not quasi-reflexive.

Proof. It is clear that we may find in $X^{* *}$ a separable closed subspace $V$, $V \subset X$, and a separable normed subspace $Z$, such that the dimensions of $V / X$ and $Z$ be infinite and $Z \cap V=\{0\}$. We apply Theorem 3 replacing $X$ by $X^{*}$ and $U$ by $X$, thus obtaining a double sequence $\left(x_{m n}\right)$ with the properties there mentioned. Besides, having Lemma 3 in mind, such a double sequence can be chosen so that

$$
y_{11}, y_{12}, y_{21}, \ldots, y_{1 n}, y_{2(n-1)}, \ldots, y_{(n-1) 2}, y_{n 1}, \ldots
$$

be $w^{*}$-basic and boundedly complete. Then, if we write

$$
y_{11}, y_{12}, y_{21}, \ldots, y_{1 n}, y_{2(n-1)}, \ldots, y_{(n-1) 2}, y_{n 1}, \ldots
$$

in the form $\left(x_{n}\right)$, we have that $\left(x_{n}\right)$ is a boundedly complete $\mathrm{w}^{*}$-basic sequence of $X^{*}$ satisfying properties 1 and 2 .

The following results are proved in [3]: c) Let $X$ be a Banach space. If $X^{*}$ contains a norming closed subspace of infinite codimension, then $X$ has a basic sequence that is not $k$-shrinking for any non-negative integer $k$. d) If $X$ is a non-quasi-reflexive Banach space, then $X$ contains a closed subspace $Y$ such that $Y$ and $X / Y$ are not quasi-reflexive. Notice that result c) may be obtained from Corollary 3. On the other hand, result d) is an easy consequence of Corollaries 3 and 4.

If in Lemma 3 we take $u_{m n}=u_{n}, m, n \in \mathbb{N}$, we may obtain the following result which is a refinement of a theorem of [10]. e) Let $X$ be a separable Banach space. In $X^{*}$, let $\left(u_{n}\right)$ be a sequence that weak*converges to the origin such that

$$
\inf \left\{\left\|u_{n}\right\|: n \in N\right\}>0 .
$$


Then there is a boundedly complete $w^{*}$-basic subsequence $\left(u_{n_{j}}\right)$ of $\left(u_{n}\right)$ such that $\left[u_{n_{j}}\right]$ is 1-norming with respect to $X /\left[u_{n_{j}}\right]_{\perp}$. From the same lemma we may also oistain the following result of [10]: f) Let $X$ be a Banach space with $X^{*}$ separable. In $X^{*}$, let $\left(u_{n}\right)$ be a sequence that weak ${ }^{*}$-converges to the origin so that

$$
\inf \left\{\left\|u_{n}\right\|: n \in \mathbb{N}\right\}>0 .
$$

Then there is a boundedly complete $w^{*}$-basic subsequence $\left(u_{n_{j}}\right)$ of $\left(u_{n}\right)$. The following theorem can also be found in $[10]: g)$ Let $X$ be a separable Banach space such that there is in $X^{*}$ a closed subspace $Y$ isomorphic to a subspace of a separable conjugate space. Then $X$ has a quotient with a shrinking basis; moreover, this basis may be chosen with its biorthogonal functionals lying in $Y$, hence $Y$ has a weak*-closed subspace with a boundedely complete basis.

In the coming proposition we obtain a result which uniffes both results $f$ ) and $g$ ).

Proposition 3. Let $X$ be a separable Banach space. Let $\left(u_{n}\right)$ be a sequence in $X^{*}$ which weak ${ }^{*}$-converges to the origin and such that inf $\left\{\left\|u_{n}\right\| ; n \in \mathbb{N}\right\}>0$. If $\left[u_{n}\right]$ is isomorphic to a subspace of a separable conjugate space, then there is a $w^{*}$-basic subsequence $\left(u_{n_{j}}\right)$ of $\left(u_{n}\right)$ that is boundedly complete.

Proof. Let $Y$ a Banach space with separable dual $Y^{*}$ such that there is an isomorphism $\psi$ from $\left[u_{n}\right]$ into $Y^{*}$. Since both $X$ and $Y$ are separable, we may assume that $\left(u_{n}\right)$ is w $^{*}$-basic and that $\left(\psi\left(u_{n}\right)\right)$ is weak*-Cauchy in $Y^{*}$. Let $v_{0}$ be the weak ${ }^{*}$ limit of $\left(\psi\left(u_{n}\right)\right)$ in $Y^{*}$. Let us suppose that $v_{0}$ is distinct from the origin and contained in $\left[\psi\left(u_{n}\right)\right]$. Since $\left(\psi\left(u_{n}\right)\right)$ is a Schauder basis of $\left\{\psi\left(u_{n}\right)\right\}$, we have in this space that

$$
v_{0}=\sum_{n=1}^{\infty} a_{n} \psi\left(u_{n}\right) \text {. }
$$

If $r$ is a positive integer such that $a_{r} \neq 0$, then $v_{0}$ does not belong to the closed linear span of $\left(\psi\left(u_{n}\right)\right)_{n=r+1}^{\infty}$. Consequently, we may assume to have taken $\left(u_{n}\right)$ so that $v_{0}$ is not in $\left[\psi\left(u_{n}\right)\right]$.

If $v_{0}=0$, we write $Z:=Y, v_{n}:=\psi\left(u_{n}\right), n=1,2, \ldots$, and $\varphi$ for the identity in $Y^{*}$. If $v_{0} \neq 0$, then we write $Z$ to denote the linear subspace 
in $Y$ orthogonal to $\left\{v_{0}\right\}$ and if $L:=\operatorname{lin}\left\{v_{0}\right\}$, then $\varphi$ will denote the canonical mapping from $Y^{*}$ onto $Y^{*} / L=Z^{*}$; we write in this case $v_{n}:=\varphi\left(\psi\left(u_{n}\right)\right), n=1,2, \ldots$ In any case, whether $v_{0}$ be zero or not, we have that $\Phi=\varphi \circ \psi$ is an isomorphism from $\left[u_{n}\right]$ into $Z^{*}$ such that $\Phi\left(u_{n}\right)=v_{n}, n=1,2, \ldots$ and $\left(v_{n}\right)$ converges to the origin in $Z^{*}$ for the weak* topology.

We take now a subsequence $\left(u_{n_{j}}\right)$ of $\left(u_{n}\right)$ such that $\left(v_{n_{j}}\right)$ is a boundedly complete $w^{*}$-basic subsequence in $Z^{*}$. We choose an arbitrary sequence $\left(b_{n}\right)$ in $K$ such that $\left(\sum_{j=1}^{m} b_{j} u_{n_{j}}\right)_{m=1}^{\infty}$ be bounded in $X^{*}$. Then

$$
\left(\sum_{j=1}^{m} b_{j} \Phi\left(u_{n_{j}}\right)\right)_{m=1}^{\infty}=\left(\sum_{j=1}^{m} b_{j} v_{n_{j}}\right)_{m=1}^{\infty}
$$

is bounded in $Z^{*}$ and, consequently, $\sum_{j=1}^{\infty} b_{j} v_{n_{j}}$ converges in this space. On the other hand, $\sum_{j=1}^{\infty o} b_{j} u_{n_{j}}$ converges in $X^{*}$ and the conclusion follows.

As a consequence of result $g$ ), it is shown in [10] that if $X$ is separable Banach space and $Y$ is a subspace of $X^{*}$ isomorphic to $l^{1}$, then $X$ has a quotient isomorphic to $c_{0}$. In the next proposition we obtain a refinement of this result.

Proposition 4. Let $X$ be a Banach space and let $\left(u_{n}\right)$ be a sequence in $X^{*}$ equivalent to the unit vector basis of $l_{1}$. If $\left(u_{n}\right)$ is weak $k^{*}$-Cauchy then $X /\left[u_{n}\right]_{\perp}$ is isomorphic to $c_{0}$.

Proof. Let $u$ be the weak ${ }^{*}$ limit of $\left(u_{n}\right)$. If $u$ happens to be the origin of $X^{*}$, then we proceed as follows. For each $x$ in $X$, we define $T x:=$ $\left(\left\langle x, u_{n}\right\rangle\right)$. Then $T$ is a continuous linear mapping from $X$ into $c_{0}$. We also have that $T^{*}: l^{1} \longrightarrow X^{*}$ is weak ${ }^{*}$-weak* continuous and, for each $\left(a_{n}\right)$ of $l^{1}, T^{*}\left(\left(a_{n}\right)\right)=\sum_{n=1}^{\infty} a_{n} u_{n}$. If $\left(a_{n}\right)$ and $\left(b_{n}\right)$ are distinct elements of $l^{1}$, it is clear that $T^{*}\left(\left(a_{n}\right)\right) \neq T^{*}\left(\left(b_{n}\right)\right)$ and so $T(X)$ is dense in $c_{0}$. It then follows that $T^{*}\left(l_{1}\right)=\left\{u_{n}\right\}$ and $T^{*}\left(B\left(l_{1}\right)\right)$ is a neighborhood of the origin in $\left[u_{n}\right]$, hence there is a positive integer $m$ such that $m T^{*}\left(B\left(l_{1}\right)\right)$ contains $B\left(X^{*}\right) \cap T^{*}\left(l_{1}\right)$. Let $\left\{v_{j}: j \in J, \geq\right\}$ be a net in $B\left(X^{*}\right) \cap T^{*}\left(l_{1}\right)$ that weak ${ }^{*}$-converges to an element $v$ of $X^{*}$. Since $T^{*}\left(B\left(l_{1}\right)\right)$ is weak ${ }^{*}$ compact, $v$ belongs to $m T^{*}\left(B\left(l_{1}\right)\right) \subset T^{*}\left(l_{1}\right)$, from where we deduce that 
$B\left(X^{*}\right) \cap T^{*}\left(l_{1}\right)$ is weak ${ }^{*}$-closed in $X^{*}$ and, making use of Krein-Smulian's Theorem, $\left.[9], T^{*}\left(l_{1}\right)\right)$ is weak ${ }^{*}$-closed in $X^{*}$. We now obtain after [13] that $T$ is onto and the result follows.

Let us assume now that $u$ is not the origin of $X^{*}$. Let $Y$ be the subspace of $X$ orthogonal to $\{u\}$ and let $L$ be the linear span of $\{u\}$. If $u$ does not belong to $\left[u_{n}\right]$ and $\varphi$ denctes the canonical mapping from $X^{*}$ onto $Y^{*}=X^{*} / L$, it follows that $\varphi:\left[u_{n}\right] \longrightarrow Y^{*}$ is one-to-one and $\left.\varphi\left(u_{n}\right)\right)$ is a sequence in $Y^{*}$ which is equivalent to the unit basis of $l_{1}$ and such that it weak* converges to zero. Hence we have that $Y /\left|\varphi\left(u_{n}\right)\right|_{\perp}$ is isomorphic to $c_{0}$. On the other hand, $\left[\varphi\left(u_{n}\right)\right]_{\perp}=\left[u_{n}\right]_{\perp}$ and $Y /\left[u_{n}\right]_{\perp}$ is a closed hyperplane of $X /\left[u_{n}\right]_{\perp}$, thereby isomorphic to $c_{0}$. Finally, if $u$ is in $\left[u_{n}\right]$, there is $\left(a_{n}\right) \in l^{1}$ such that $u=\sum_{n=1}^{\infty} a_{n} u_{n}$ in $X^{*}$. Let $r$ be a positive integer such that $a_{r} \neq 0$. We set $v_{j}:=u_{j}, j=1,2, \ldots, r-1$, $v_{j}:=u_{j+1}, j=r, r+1, \ldots$, . Then, $\left(v_{n}\right)$ is equivalent to the unit vector basis of $l^{1}$ and thus $u$ is not in $\left|v_{n}\right|$, and hence, applying what we said above, $X /\left[v_{n}\right]_{\perp}$ is isomorphic to $c_{0}$.

Proposition 5. Let $X$ be a Banach space that contains no copy of $l^{1}$. If $X^{*}$ contains a copy of $l^{1}$, then $X$ has a quotient which is isomorphic to $c_{0}$.

Proof. We know after [8] that $X^{*}$ contains a sequence $\left(u_{n}\right)$, equivalent to the unit vector basis of $l^{1}$, which is a Cauchy sequence for the weak* topology, hence it suffices to make use of the former proposition to reach the conclusion.

Note. If in Lemma 2 we take $x_{m n}=x_{n}, m, n \in \mathbb{N}$, and $X^{*}$ is separable and such that $\left\{u_{1}, u_{2}, \ldots, u_{n}, \ldots\right\}$ be dense in $X^{*}$, then the following result follows, [10]: h) In the Banach space $X$, let $\left(x_{n}\right)$ be a sequence that converges weakly to the origin. If $X^{*}$ is separable and in $f\left\{x_{n} \|: n \in\right.$ $\mathbb{N}\}>0$, then there is a subsequence $\left(y_{n}\right)$ of $\left(x_{n}\right)$ such that it is basic and shrinking. On the otber hand, if in Lemma 2 we take $x_{m n}=x_{n}$, $m, n \in \mathbb{N},\left(x_{n}\right)$ having a weak ${ }^{*}$-adherent point $x_{0}$ in $X^{* *}, x_{0} \notin X$, $X^{*}$ is separable $\left\{u_{1}, u_{2}, \ldots, u_{n}, \ldots\right\}$ is a dense subset of the hyperplane $H$ orthogonal to $x_{0}$, then we obtain, by renorming $X$ if necessary so 
that $\left[u_{n}\right]$ be 1-norming, the following result, [20]: i) Let $\left(x_{n}\right)$ be a bounded sequence in the Banach space $X$ such that $\left\{x_{1}, x_{2}, \ldots, x_{n}, \ldots\right\}$ is not weakly relatively compact. If $X^{*}$ is separable, then there is a subsequence $\left.y_{n}\right)$ of $\left(x_{n}\right)$ such that if $\left(z_{n}\right)$ is an arbitrary subsequence of $\left(y_{n}\right)$ then $\left(z_{n}\right)$ is a 1 -shrinking basis of $\left[z_{n}\right]$.

The following proposition is an isometric version of a result of [10].

Proposition 6. In the Banach space $X$, let $\left(u_{n}\right)$ be a sequence in $X^{*}$ converging weakly to the origin such that $\left[u_{n}\right]^{*}$ is separable and inf $\left\{\left\|u_{n}\right\|: n \in N\right\}>0$. Then, there is a subsequence $\left(u_{n_{j}}\right)$ of $\left(u_{n}\right)$, $w^{*}$-basic and shrinking, such that $\left[u_{n}\right]^{*}$ is isometric to $X /\left[\left.u_{n_{j}}\right|_{\perp}\right.$.

Proof. Since $\left[u_{n}\right]^{*}$ is separable, we apply result $h$ ) to extract a subsequence of $\left(u_{n}\right)$, which we shall still keep denoting by $\left(u_{n}\right)$, that is basic and shrinking. We take a dense subset $\left\{v_{1}, v_{2}, \ldots, v_{n}, \ldots\right\}$ of $B\left(\left|u_{n}\right|\right)$. For each pair of positive integers $m$ and $n$, let $x_{m n}$ be an element of $X$ such that

$$
\left\|x_{m n}\right\|=\left\|v_{n}\right\| \text { and }\left|\left\langle x_{m n}, v_{n}\right\rangle\right|>\left\|v_{n}\right\|-\frac{1}{m} .
$$

We put $Y:=\left[x_{m n}\right]$. Then $Y$ is a 1 -norming subspace of $X^{*}$ respect to $\left[u_{n}\right]$. We apply Lemma 2 to obtain a subsequence $\left(u_{n_{f}}\right)$ of $\left(u_{n}\right)$ so that if $\varphi$ is the canonical mapping from $X^{* *}$ onto $\left[u_{n_{j}}\right]^{\perp}=\left[u_{n_{j}}\right]$ it follows that

$$
\stackrel{\circ}{B}\left(\left[u_{n_{j}}\right]\right)=\stackrel{\circ}{B}\left(\left[u_{n_{j}}\right]^{*}\right)=\varphi(\stackrel{\circ}{B}(X))=\varphi\left(\stackrel{\circ}{B}\left(X^{* *}\right)\right),
$$

from where we deduce that $\left(u_{n_{j}}\right)$ is $w^{*}$-basic and $\left[u_{n_{j}}\right]^{*}$ is isometric to $X /\left[u_{n_{j}}\right]_{\perp}$. It is plain that $\left(u_{n_{j}}\right)$ is shrinking.

The following results can be found in [19]: j) Let $\left(x_{n}\right)$ be a bounded sequence in the Banach space $X$. If $\left\{x_{n}: n \in I N\right\}$ is not weakly relatively compact then there is a subsequence $\left(y_{n}\right)$ of $\left(x_{n}\right)$ such that every subsequence $\left(z_{n}\right)$ of $\left(y_{n}\right)$ is basic and has Property $P^{*}$. k) If $X$ is a separable non-reflexive Banach space, then $X$ has a quotient which has a basis of the type $P$.

In what follows we shall obtain properties related with results $j$ ) and k). 
Proposition 7. In a Banach space $X$, let $\left(z_{m n}\right)_{n=1}^{\infty}$ be a bounded sequence which has in $X^{* *}$ a weak ${ }^{*}$-adherent point $x_{m}, m=1,2, \ldots, r$, such that $x_{1}, x_{2}, \ldots, x_{r}$ are linearly independent and $X \cap \operatorname{lin}\left\{x_{1}, x_{2}, \ldots, x_{r}\right\}=$ $\{0\}$. Then, there is a subsequence $\left(y_{m n}\right)_{n=1}^{\infty}$ of $\left(z_{m n}\right)_{n=1}^{\infty}, m=1,2, \ldots, r$, so that

$$
y_{11}, y_{21}, \ldots, y_{r 1}, \ldots, y_{1 n}, y_{2 n}, \ldots, y_{r n}, \ldots
$$

is a basic sequence in $X$ with Property $P^{*}$.

Proof. If we denote by $\left(x_{m n}\right)_{n=1}^{\infty}$ the subsequence of $\left(z_{m n}\right)_{n=1}^{\infty}$ formed by all those elements with norms greater than $\frac{1}{2}\left\|x_{m}\right\|$, it follows that $\left(x_{m n}\right)_{n=1}^{\infty}$ has $x_{m}$ as a weak ${ }^{*}$-adherent point in $X^{* *}$ and

$$
\text { in } f\left\{\left\|x_{m n}\right\|: n \in \mathbb{N}\right\}>0, \quad m=1,2, \ldots, r .
$$

We write $x_{m n}:=x_{r n}, x_{m}:=x_{r}, m=r+1, r+2, \ldots, n \in \mathbb{N}$. We then have $\left[x_{n}\right]=\operatorname{lin}\left\{x_{1}, x_{2}, \ldots, x_{r}\right\}$ and so the subspace of $X^{*}$ orthogonal to $\left[x_{n}\right]$ is norming. We apply Proposition 1 and obtain a subsequence $\left(u_{m n}\right)_{n=1}^{\infty}$ of $\left(x_{m n}\right)_{n=1}^{\infty}, m \in \mathbb{N}$ such that

$$
\begin{gathered}
u_{11}-x_{1}, u_{12}-x_{1}, u_{21}-x_{2,}, \cdot, u_{1 n}-x_{1}, u_{2(n-1)}-x_{2,}, u_{(n-1) 2}-x_{n-1}, \\
u_{n 1}-x_{n}, \ldots
\end{gathered}
$$

is a basic sequence in $X^{* *}$ and

$$
u_{11}, u_{12}, u_{21}, \ldots, u_{1 n}, u_{2(n-1)}, \ldots, u_{(n-1) 2}, u_{n 1}, \ldots
$$

is a basic sequence in $X$. If we write $y_{m n}:=u_{m(r n)}, m=1,2, \ldots, r$, $n \in \mathbb{N}$, we have that

$$
y_{11}-x_{1}, y_{21}-x_{2}, \ldots, y_{r 1}-x_{r}, \ldots, y_{1 n}-x_{1}, y_{2 n}-x_{2}, \ldots, y_{r n}-x_{r}, \ldots
$$

is a basic sequence in $X^{*}$ and

$$
y_{11}, y_{21}, \ldots, y_{r 1}, \ldots, y_{1 n}, y_{2 n}, \ldots, y_{r n}, \ldots
$$

is a basic sequence in $X$. Let $F$ the closed linear span of (18) in $X^{* *}$ and let $G$ be the closed linear subspace in $X$ spanned by (19). We take $z$ in $F \cap X$. Then

$$
z=\sum_{n=1}^{\infty} \sum_{m=1}^{\infty} a_{m n}\left(y_{m n}-x_{m}\right)
$$


Let $T$ be the linear projection on $X+\operatorname{lin}\left\{x_{1}, x_{2}, \ldots, x_{r}\right\}$ over lin $\left\{x_{1}, x_{2}, \ldots, x_{r}\right\}$ along $X$. Since $T$ is continuous, it follows that

$$
\begin{gathered}
0=T x=\sum_{n=1}^{\infty} \sum_{m=1}^{r} a_{m n} T\left(y_{m n}-x_{m}\right)=-\sum_{n=1}^{\infty} \sum_{m=1}^{r} a_{m n} x_{m}= \\
-\sum_{m=1}^{r}\left(\sum_{n=1}^{\infty} a_{m n}\right) x_{m}
\end{gathered}
$$

and hence

$$
\sum_{n=1}^{\infty} a_{m n}=0, \quad n=1,2, \ldots, r, \quad z=\sum_{n=1}^{\infty} \sum_{m=1}^{r} a_{m n} y_{m n},
$$

from where we have that $F \cap X$ is contained in $G$. Suppose now that our formerly considered vector $z$ has the form $\sum_{m=1}^{r} b_{m} y_{m 1}$. Then

$$
\sum_{m=1}^{r} b_{m} y_{m 1}=\sum_{n=1}^{\infty} \sum_{m=1}^{r} a_{m n} y_{m n}
$$

and so

$$
a_{m n}=0, \quad n=2,3, \ldots, \quad b_{m}=a_{m 1}=\sum_{n=1}^{\infty} a_{m n}=0, \quad m=1,2, \ldots, r .
$$

Consequently, if $E$ is the linear span of $\left\{y_{m 1}: m=1,2, \ldots, r\right\}$ it follows that $E \cap F=\{0\}$. For a given integer $m \leq r$, we have that $y_{m 1} \in E$ and, for $n>1$,

$$
\begin{gathered}
y_{m n}=y_{m 1}+\left(y_{m 2}-y_{m 1}\right)+\cdot+\left(y_{m n}-y_{m(n-1)}\right)=y_{m 1} \\
+\left(y_{m 2}-x_{m}-\left(y_{m 1}-x_{m}\right)\right)+\ldots \\
+\left(y_{m n}-x_{m}-\left(y_{m(n-1)}-x_{m}\right)\right) \in E+F \cap X
\end{gathered}
$$

and hence, $G=E+F \cap X$. We now write the sequence (19) in the form $\left(z_{n}\right)$. For each positive integer $n$, we put $p(n)$ and $q(n)$ to denote the positive integers such that $z_{n}=y_{p(n) q(n)}$. Given an arbitrary element $x$ of $G$, it may be written in a unique way as $x=y+z, y \in E, z \in F \cap X$. Then

$$
z=\sum_{n=1}^{\infty} c_{n}\left(z_{n}-x_{p(n)}\right), \sum_{n=1}^{\infty} c_{n}=0
$$


and since $z_{1}, z_{1}-z_{2}, \ldots, z_{r-1}-z_{r}$ is a basis for $E$, we have that

$$
y=b_{1} z_{1}+b_{2}\left(z_{1}-z_{2}\right)+\ldots+b_{r}\left(z_{r-1}-z_{r}\right) .
$$

We write

$$
\kappa_{n}:=\sum_{j=1}^{n} c_{j}, \quad n=1,2, \ldots
$$

Then

$$
z=\lim _{m} \sum_{n=1}^{m} c_{n} z_{n}=\lim _{m}\left(\sum_{n=1}^{m} \kappa_{n}\left(z_{n}-z_{n+1}\right)+\kappa_{m+1} z_{m+1}\right)
$$

and, since $\left(\kappa_{m+1} z_{m+1}\right)_{m=1}^{\infty}$ converges to the origin, it then follows that

$$
z=\sum_{n=1}^{\infty} \kappa_{n}\left(z_{n}-z_{n+1}\right)
$$

From here we deduce that

$x=y+z=b_{1} z_{1}+\left(b_{1}+\kappa_{1}\right)\left(z_{1}-z_{2}\right)+\ldots+\left(b_{r}+\kappa_{r-1}\right)\left(z_{r-1}-z_{r}\right)+$

$$
\sum_{n=r}^{\infty} \kappa_{n}\left(z_{n}-z_{n+1}\right)
$$

and therefore, if we set

$$
v_{1}:=z_{1}, \quad v_{n+1}:=z_{n}-z_{n+1}, \quad n=1,2, \ldots,
$$

it is immediate that $\left(v_{n}\right)$ is a Schauder basis in $\left[v_{n}\right]=\left[z_{n}\right]$, and the result now follows.

Theorem 5. Let $X$ be a Banach space with $X^{* *}$ separable. Let $r$ be a positive integer. If the dimension of $X^{* *} / X$ is greater or equal than $r$, then there is a closed subspace $Y$ of $X$ such that $X / Y$ is a quasireflexive Banach space of order $r$ with a Schauder basis that is shrinking and satisfies Property $P$.

Proof. According to Corollary 2, we may assume that $X$ is quasireflexive of order $r$. Let $M$ be the subspace of $X^{* * *}$ orthogonal to $X$. In 
$M$ we take a basis $\left\{x_{1}, x_{2}, \ldots, x_{r}\right\}$. We find in $X^{*}$ a sequence $\left(x_{m n}\right)_{n=1}^{\infty}$ that converges to $x_{m}$ in $X^{* * *}$ for the weak topology, $m=1,2, \ldots, r$. We proceed as in the proof of last proposition to obtain a subsequence $\left(y_{m n}\right)_{n=1}^{\infty}$ of $\left(x_{m n}\right)_{n=1}^{\infty}, m=1,2, \ldots, r$, such that

$$
y_{11}, y_{21}, \ldots, y_{r 1}, \ldots, y_{1 n}, y_{2 n}, \ldots, y_{r n}, \ldots
$$

be a basic sequence in $X^{*}$ with Property $P^{*}$. On the other hand, having in mind Lemma 3, we may obtain (21) so that it is $w^{*}$-basic and boundedly complete. Then, if $Y$ is the subspace of $X$ orthogonal to the linear span of (21), it follows that $X / Y$ is quasi-reflexive of order $r$, has a shrinking Schauder basis and, after result a), such basis satisfies Property $P$.

The anthor wishes to express his gratitude to the referee for several interesting suggestions concerning references [4], [5], [6] and [7] in particular, which will undoubtedly help the reader to gain a better understanding of this material, and also for a few remarks on some results which have certainly improved the final version of the paper.

\section{References}

[1] BESSAGA, C. and PELCZYNSKI, A., On bases and unconditional convergence of series in Banach spaces, Bull. Acad. Pol. Sci. 4, 379383 (1959).

[2] DAVIS, W. J. and JOHNSON, W. B., A renorming of non-reflexive Banach spaces, Proc. Amer. Math. Soc. 37, 486-488 (1973).

[3] DAVIS, W. J, and JOHNSON, W. B., Basic sequences and norming subspaces in non-quasi-reflexive Banach spaces, Israel J. Math. 14, 352-367 (1973).

[4] DEville, R., GODEFroY, G., HARE, D. and ZIZLER, V., Phelps spaces and finite-dimensional decompositions, Bull. Austral. Math. Soc. 37,2, 263-271 (1988).

[5] FINET, C., Subspaces of Asplund Banach spaces with the point of continuity property, Israel J. of Math. 60,2, 191-198 (1987). 
[6] FINET, C., Basic sequences and smooth norms in Banach spaces, Studia Math. 89, 1-9 (1988).

[7] GHOUSSOUB, G. and MAUREY, B., A nonlinear method for constructing certain basic sequences in Banach spaces, Illinois $J$. of Math. 34, 607-613 (1990).

[8] HAGLER, J. and JOHNSON, W. B., On Banach spaces whose dual balls are not weak* sequentially compact, Israel J. Math. 28, 325330 (1977).

[9] HORVÁTH, J., Topological Vector Spaces and Distributions I, Reading, Massachussets, 1966.

[10] JOHNSON, W. B. and ROSENTHAL, H. P., On $w^{*}$-basic sequences and their applications to the study of Banach spaces, Studia Math. 43, 77-92 (1972).

[11] KADEC, M. I., On the connection between weak and strong convergence, Dopovidi Akad. Nauk Ukrain 9, 949-952 (1959).

[12] KLEE, V. L., Mappings into normed linear spaces, Fund. Math. 49, 25-34 (1961).

[13] KÖTHE, G., Topological Vector Spaces I, Springer, BerlinHeidelberg-New York, 1979.

[14] PELCZYNSKI, A., A note on the paper of I. Singer "Basic sequences and reflexivity of Banach spaces", Studia Math. 21, 372374 (1962).

[15] SINGER, I., Basic sequences and reflexivity of Banach spaces, Studia Math. 21, 351-369 (1962).

[16] SINGER, 1., Bases in Banach Spaces I, Springer, BerlinHeidelberg-New York, 1970.

[17] VALDIVIA, M., Bases y casi-reflexividad en espacios de Banach, Rev. Real Acad. Ciencias Exactas, Fisicas y Naturales de Madrid 82, 45-53 (1988). 
[18] VALDIVIA, M., Banach spaces $X$ with $X^{* *}$ separable, Israel $J$. Math. 59, 107-111 (1987).

[19] VALDIVIA, M, A characterization of Totally Reflexive Frchet Spaces, Math. Z. 200, 327-346 (1989).

[20] VALDIVIA, M., On basic sequences in Banach spaces, Note di Matematica 22, 245-258 (1992).

Departamento de Análisis Matemático

Universidad de Valencia

Dr. Moliner, 50

46100 Burjasot (Valencia)

Recibido: 8 de Enero de 1997

Spain

Revisado: 28 de Abril de 1997 